\title{
A Hierarchical Scheme for Geodesic Anatomical Labeling of Airway Trees
}

\author{
Aasa Feragen ${ }^{1}$, Jens Petersen ${ }^{1}$, Megan Owen $^{2}$, Pechin Lo ${ }^{1,3}$, \\ Laura H. Thomsen ${ }^{4}$, Mathilde M.W. Wille ${ }^{4}$, \\ Asger Dirksen ${ }^{4}$, and Marleen de Bruijne ${ }^{1,5}$
}

1 Department of Computer Science, University of Copenhagen, Denmark

2 David R. Cheriton School of Computer Science, University of Waterloo, Canada

3 Department of Radiology, University of California, Los Angeles, USA

4 Lungemedicinsk afdeling, Gentofte Hospital, Denmark

5 Biomedical Imaging Group Rotterdam, Erasmus MC, The Netherlands

\{aasa, phup, marleen\}@diku.dk, megan.owen@uwaterloo.ca

\begin{abstract}
We present a fast and robust supervised algorithm for labeling anatomical airway trees, based on geodesic distances in a geometric tree-space. Possible branch label configurations for a given tree are evaluated based on distances to a training set of labeled trees. In tree-space, the tree topology and geometry change continuously, giving a natural way to automatically handle anatomical differences and noise. The algorithm is made efficient using a hierarchical approach, in which labels are assigned from the top down. We only use features of the airway centerline tree, which are relatively unaffected by pathology.

A thorough leave-one-patient-out evaluation of the algorithm is made on 40 segmented airway trees from 20 subjects labeled by 2 medical experts. We evaluate accuracy, reproducibility and robustness in patients with Chronic Obstructive Pulmonary Disease (COPD). Performance is statistically similar to the inter- and intra-expert agreement, and we found no significant correlation between COPD stage and labeling accuracy.
\end{abstract}

Keywords: airway tree labeling, geodesic matching.

\section{Introduction}

Computed Tomography (CT) is an important tool in the analysis of diseases affecting the airways. Using image segmentation methods, three-dimensional models of the airway surfaces can be constructed, and their dimensions measured. Such measurements are, however, dependent on the location in which they are made 4], so solving the problem of finding anatomically corresponding positions in different airway trees is crucial to robustly compare measurements across patients. It can be solved by assigning anatomical names to the airway tree branches. This is nontrivial, since the topology of the anatomical airway 

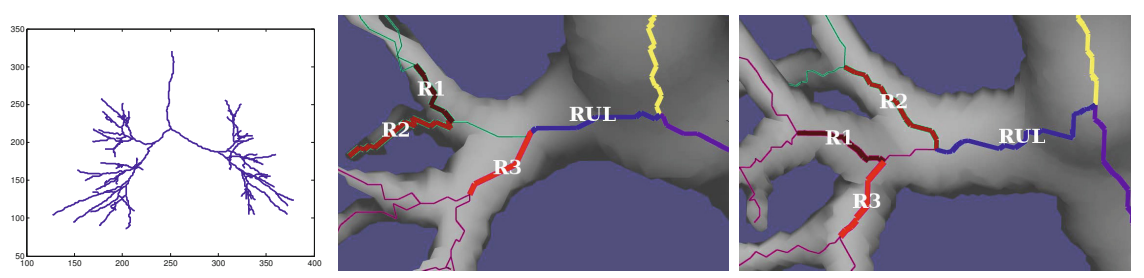

Fig. 1. Left: The method takes only the airway centerline tree as input. Middle-right: Airway trees are frequently topologically different, while geometric differences are small.

tree changes from person to person 1 , and the segmented trees have differences introduced by noise, including missing and spurious branches.

Several types of airway branch labeling algorithms have appeared previously. Van Ginneken et al. [11] and Lo et al. [5] use machine learning on branch features, with additional assumptions on airway tree topology. Among the features used are branch length, radius, orientation, cross-sectional shape and bifurcation angle. Branch length and radius are sensitive to the presence of structural noise or diseases like cystic fibrosis, tuberculosis or Chronic Obstructive Pulmonary Disease (COPD), and assumptions on tree topology make these methods vulnerable to topological differences. Tschirren et al. use association graphs [10] for pairs of airway trees, which incorporate information from both trees, such that maximal cliques in the association graph induce branch matchings between the original graphs. This is a combinatorial construction, although it can depend on the geometric properties of the initial trees. Such a separation of geometric and combinatorial properties can be problematic, as airway trees are often geometrically and visually similar despite being combinatorially different, as in Fig. 1 Feragen et al. 2] label airways based on geodesics (shortest paths) in a space of trees. Their tree-space is highly non-linear and has no known efficient algorithm for geodesic computation, making labeling of a complete airway tree infeasible.

We present a novel supervised method for automatic airway branch labeling, based on shortest paths in a space of geometric trees [1]. This tree-space is less general than that of Feragen et al. [2, but allows geodesics to be computed in polynomial time 6]. Possible label configurations on an unlabeled tree are evaluated based on distances to all trees in a training set of labeled trees. In tree-space, both topology and geometric branch attributes are allowed to change continuously, and we can thus compare trees with different topology and branch geometry. The only feature used is the airway centerline tree, see Fig. 1. The method does not depend directly on the branches identified by the segmentation, but rather on a subtree spanning the labeled branches, defined in Fig. 2, where branches may be concatenations of branches from the originally segmented tree. As a consequence, the method is less sensitive to structural noise such as false or missing branches than methods that work only on the originally segmented

1 Tree topology describes how tree branches are connected. The tree topologies in our data are plotted in http://image.diku.dk/aasa/miccai_supplemental.tar.gz 



Fig. 2. Left: Given a configuration of leaf labels on an airway centerline tree, extract the subtree spanning the leaf labels and prune off the rest, giving the subtree spanning the labels, a geometric leaf-labeled tree which can be compared to the training data. Right: After assigning a set of labels, each label is moved to the branch in the segmented airway tree closest to the root which is not part of the subtree spanning the other labels.

branches. The method is implemented in a hierarchical fashion, making it sufficiently fast to be of practical use. A thorough leave-one-patient-out evaluation of the algorithm is made on a set of 40 segmented airway trees from 20 subjects different stages of COPD (healthy-severe), labeled by 2 medical experts. We evaluate accuracy, reproducibility and robustness to disease stages.

\section{Anatomical Branch Labeling}

Airway branch labels correspond to the division of the lung into compartments: LMB and RMB lead to the left and right lungs; LUL, RUL, L4+5, R4+5, LLB, RLL lead to the lobes; and R1-R10, L1-L10 lead to (up to) 10 segments in each lung. In addition, intermediate branch names appear in the literature, whose presence depends on the topology of the anatomical airway tree. However, if the segment branch labels and the airway tree structure are known, the remaining branch labels can be reconstructed trivially. For this reason, a leaf-labeled airway tree (where the leaf labels are segment labels) is equivalent to a labeled airway tree. Thus we focus on evaluating the assignment of segment labels in this paper.

Methodology. Each airway tree was normalized by person height as an isotropic scaling parameter. Each branch was represented by 6 landmark points sampled equidistantly along the centerline, translated so that the first landmark point was placed at the origin. Thus, each branch $e$ is represented by a vector $x_{e} \in \mathbb{R}^{15}$.

For an arbitrary unlabeled airway tree $T$, we attach a set of 20 leaf labels corresponding to the 20 segmental bronchi, named $X=\{L 1, \ldots, L 10, R 1, \ldots, R 10\}$. Our training set consists of $n$ airway trees which have been labeled by two experts in pulmonary medicine. We extract the subtree spanning the labels, as defined in Fig. 2], and obtain $2 n$ leaf-labeled trees $\mathfrak{T}=\left\{T_{1}^{1}, \ldots, T_{n}^{1}, T_{1}^{2}, \ldots, T_{n}^{2}\right\}$.

Given an unlabeled airway tree $T$, we proceed as follows. Denote the set of branches in $T$ by $E$. A labeling of $T$ is a map $L: X \rightarrow E$. We only consider labelings where the leaf labels are all attached to leaves in the subtree spanning the labels, i.e., we do not consider labelings where two leaf labels are attached to branches on the same path to the root. Given such a labeling $L$, extract the subtree $T_{L}$ of $T$ spanning the labels. For each labeled tree $\tilde{T}$ in our training set and each $T_{L}$, we compute the shortest-path distance $d\left(\tilde{T}, T_{L}\right)$ between the trees 

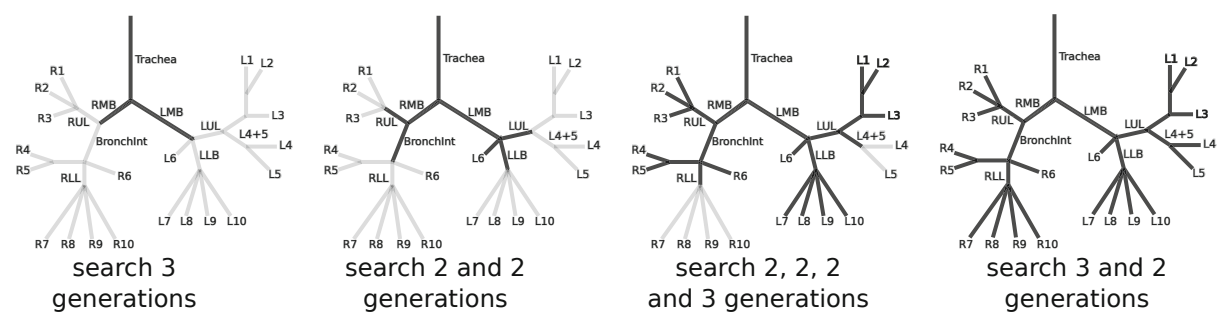

Fig. 3. Hierarchical labeling: In each step, search through 2 or 3 generations of branches (as indicated in the figure) to find an optimal alignment of a set of labels, obtaining a leaf-labeled subtree of the segmented airway tree similar to the trees shown in black. The real tree topology may differ; the figure only illustrates the stepwise hierarchy.

$\tilde{T}$ and $T_{L}$ in the tree-space defined below. We find a labeling of $T$ by choosing the labeled tree $T_{\text {labeled }}$ among the $T_{L}$ that satisfies:

$$
T_{\text {labeled }}=\underset{T_{L}}{\arg \min } \sum_{\tilde{T} \in \mathfrak{T}} d\left(\tilde{T}, T_{L}\right) .
$$

Ideally, we would search through the whole airway tree $T$, test all admissible configurations $T_{L}$ of the 20 segment leaf labels and select the one that optimizes (11). However, for an airway tree with 100 branches, the search space size is on the order of $100^{20}$, which is too large to handle. In order to ensure computational feasibility, we choose a hierarchical subtree approach, where labels of different generation are added subsequently, see Fig. 3. In the first step, 2 generations below the trachea are searched for the optimal configurations of the RMB, LMB labels. In the second step, 2 and 2 generations below the RMB and LMB, resp., are searched for the optimal configurations of $\{$ RUL, BronchInt, L6, LLB, LUL $\}$. In the third step, 2, 2, 2 and 3 generations below RUL, BronchInt, LLB, and LUL are searched for the optimal configurations of \{R1-R5, L1-L3, L4+5 L7L10 . In the final step, 3 and 2 generations below RLL, R4+5 are searched for optimal configurations of $\{\mathrm{R} 4, \mathrm{R} 5, \mathrm{R} 7-\mathrm{R} 10\}$.

In particular, we treat more shallow branches as leaves in the first steps of the algorithm, and work our way down to the segments. In each step of the hierarchical label placement, we pick the optimal branches for the given set of labels and backtrace each label through the path to the root, see Fig. 2,

Tree-Space. The tree-space used in this paper is a straight-forward generalization of the tree-space from [1, generalizing single-dimensional shape vectors on the branches to multi-dimensional ones. Any two trees are joined by a shortest path through this space, whose length defines a distance (a metric) on tree-space.

Each point in tree-space is a leaf-labeled tree, with the leaves labeled by some fixed set $X$. Each edge in a leaf-labeled tree can be represented by a partition of $X$ into the leaves descending from the edge, and the remaining leaves (including

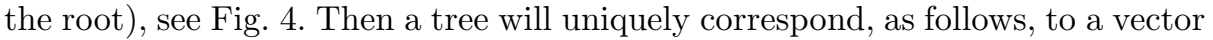
in $\left(\mathbb{R}^{15}\right)^{S}$, where $S$ is the number of possible partitions of $X$. Each consecutive set 

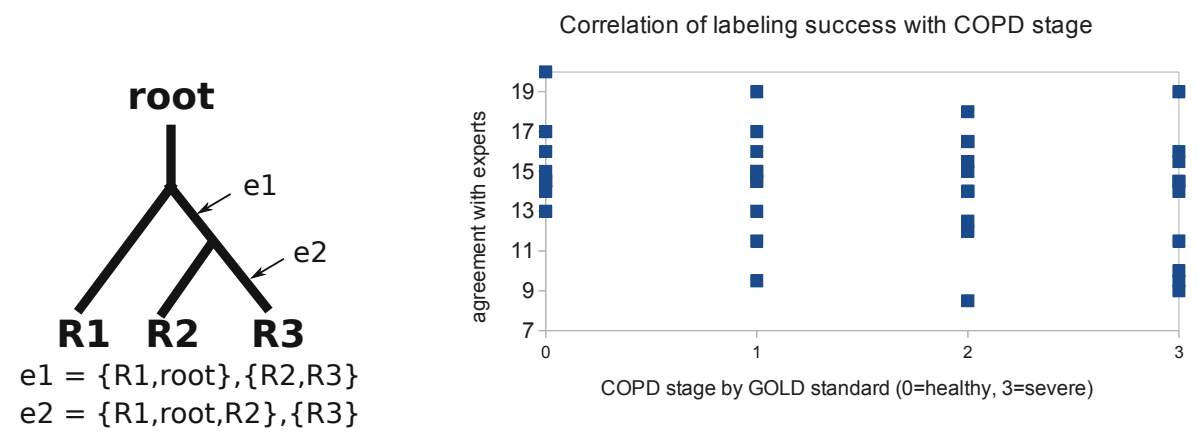

Fig. 4. Left: Tree edges are defined by partitions on the leaf label set. Right: The number of correctly assigned branch labels per segmented airway versus COPD stage.

of 15 coordinates corresponds to a possible partition of $X$. If the edge associated with that partition appears in the tree, then those 15 coordinates will be its branch vector, and otherwise they are all 0 . Certain edges can never appear in a tree together (e.g., an edge that splits $\{R 1, R 2\}$ off from the rest of the tree and an edge that splits $\{R 1, R 3\}$ off), so not all vectors are possible. Tree-space is precisely those vectors in $\left(\mathbb{R}^{k}\right)^{S}$ that correspond to trees. Thus, tree-space is a subset of Euclidean space. The shortest-path distance between two trees is the shortest path between them that remains fully within this restricted subspace, with the length of the path being measured in the ambient Euclidean space using the Euclidean metric. An analytic formula for this distance does not exist, but it can be computed recursively in polynomial time. See [6] for details and code 2

\section{Experimental Results}

Data. We work with a set of 40 airway tree centerlines obtained from low-dose (120 kV and $40 \mathrm{mAs}$ ) pulmonary CT scans from the Danish lung cancer screening trial [7. The images came from 20 subjects scanned at two different times, with an average interval of 5 years. There were 5 asymptomatic subjects and 5 from each of 3 different patient groups with mild, moderate and severe COPD according to the GOLD standard [9]. The images were segmented, centerlines extracted and branching points detected all automatically as described in [8].

The 40 airway trees were manually labeled by two experts in pulmonary medicine, who assigned segment labels $L 1-L 10$ and $R 1-R 10$ to the airway trees; the remaining labels in Fig. 3 were deduced from these. This was done using in-house developed software, simultaneously showing the segmented airway and centerline, which can be rotated, panned and zoomed, as well as a CT cross-section perpendicular to and centered on any given point of the airway.

2 Code:

http://vm1.cas.unc.edu/stat-or/webspace/miscellaneous/provan/treespace/ 
Table 1. Labeling results. Agreement with two experts measures $\%$ of agreement of the automatic labeling with the experts' labeling in the cases where the experts agree. Average intra-expert agreement and Automatic reproducibility measure the reproducibility of the experts' and automatic labelings, respectively, on pairs of scans of the same patient. The automatic method does not always assign all labels; this happens when the search subtree does not have sufficiently many leaves. Plots of airway trees with attached labels as well as tables with the complete branch labeling can be found at http://image.diku.dk/aasa/miccai_supplemental.tar.gz

\begin{tabular}{|c|c|c|c|c|c|c|c|c|}
\hline $\begin{array}{l}\overrightarrow{0} \\
\overrightarrow{\widetilde{J}} \\
\stackrel{\Xi}{\mid}\end{array}$ & 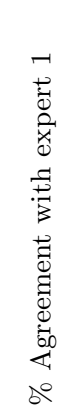 & 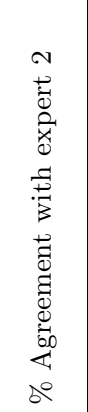 & 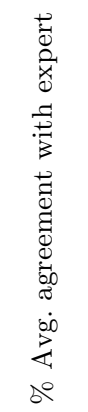 & 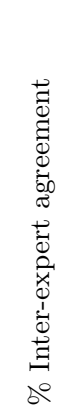 & 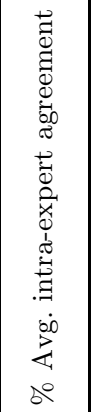 & 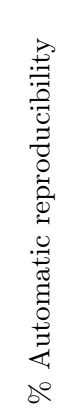 & 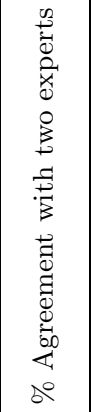 & 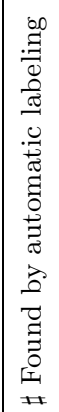 \\
\hline $\mathrm{R} 1$ & 84.62 & 69.23 & \begin{tabular}{|l|}
76.92 \\
\end{tabular} & 80.00 & \begin{tabular}{|l|}
92.50 \\
\end{tabular} & 80.00 & \begin{tabular}{|l|}
81.25 \\
\end{tabular} & 39 \\
\hline $\mathrm{R} 2$ & 82.05 & 74.36 & 78.21 & 75.00 & \begin{tabular}{|l|}
95.00 \\
\end{tabular} & 85.00 & \begin{tabular}{|l|}
90.00 \\
\end{tabular} & 39 \\
\hline R3 & 84.62 & 79.49 & 82.05 & 85.00 & \begin{tabular}{|l|}
95.00 \\
\end{tabular} & 85.00 & \begin{tabular}{|l|}
85.29 \\
\end{tabular} & 39 \\
\hline $\mathrm{R} 4$ & 92.50 & 80.00 & 86.25 & 87.50 & \begin{tabular}{|l|}
82.50 \\
\end{tabular} & 90.00 & \begin{tabular}{|l|}
91.43 \\
\end{tabular} & 40 \\
\hline $\mathrm{R} 5$ & 92.50 & 82.50 & 87.50 & 87.50 & 82.50 & 90.00 & \begin{tabular}{|l|}
94.29 \\
\end{tabular} & 40 \\
\hline $\mathrm{R} 6$ & 100.00 & 92.50 & 96.25 & 97.50 & 85.00 & 95.00 & \begin{tabular}{|l|}
94.87 \\
\end{tabular} & 40 \\
\hline$\overline{\mathrm{R} 7}$ & 60.53 & 92.11 & 76.32 & 60.00 & \begin{tabular}{|l|}
82.50 \\
\end{tabular} & 85.00 & \begin{tabular}{|l|}
91.67 \\
\end{tabular} & 38 \\
\hline$\overline{\mathrm{R} 8}$ & 39.47 & 84.21 & 61.84 & 45.00 & \begin{tabular}{|l|}
60.00 \\
\end{tabular} & 65.00 & \begin{tabular}{|l|}
77.78 \\
\end{tabular} & 38 \\
\hline$\overline{\mathrm{R} 9}$ & 52.63 & 68.42 & 60.53 & 52.50 & \begin{tabular}{|l|}
57.50 \\
\end{tabular} & 60.00 & \begin{tabular}{|l|}
76.19 \\
\end{tabular} & 38 \\
\hline R10 & 50.00 & 60.53 & 55.26 & 52.50 & \begin{tabular}{|l|}
55.00 \\
\end{tabular} & 65.00 & \begin{tabular}{|l|}
66.67 \\
\end{tabular} & 38 \\
\hline L1 & 85.00 & 72.50 & 78.75 & 67.50 & 57.50 & 75.00 & 96.30 & 40 \\
\hline$\overline{\mathrm{L} 2}$ & 85.00 & 75.00 & 80.00 & 75.00 & \begin{tabular}{|l|}
57.50 \\
\end{tabular} & 75.00 & \begin{tabular}{|l|}
93.33 \\
\end{tabular} & 40 \\
\hline L3 & 82.50 & 80.00 & 81.25 & 70.00 & \begin{tabular}{|l|}
70.00 \\
\end{tabular} & 70.00 & \begin{tabular}{|l|}
96.43 \\
\end{tabular} & 40 \\
\hline $\mathrm{L} 4$ & 65.00 & 65.00 & 65.00 & 95.00 & \begin{tabular}{|l|}
92.50 \\
\end{tabular} & 55.00 & \begin{tabular}{|l|}
68.42 \\
\end{tabular} & 40 \\
\hline L5 & 65.00 & 65.00 & 65.00 & 95.00 & 95.00 & 60.00 & \begin{tabular}{|l|}
68.42 \\
\end{tabular} & 40 \\
\hline L6 & 100.00 & 100.00 & 100.00 & 100.00 & 100.00 & 95.00 & 100.00 & 40 \\
\hline L7 & 50.00 & 65.00 & 57.50 & 42.50 & \begin{tabular}{|l|}
47.50 \\
\end{tabular} & 80.00 & \begin{tabular}{|l|}
76.47 \\
\end{tabular} & 40 \\
\hline$\overline{\mathrm{L} 8}$ & 50.00 & 62.50 & \begin{tabular}{|l|}
56.25 \\
\end{tabular} & 47.50 & \begin{tabular}{|l|}
50.00 \\
\end{tabular} & 80.00 & \begin{tabular}{|l|}
73.68 \\
\end{tabular} & 40 \\
\hline L9 & 55.00 & 50.00 & 52.50 & 50.00 & 50.00 & 65.00 & \begin{tabular}{|l|}
70.00 \\
\end{tabular} & 40 \\
\hline L10 & 55.00 & 60.00 & 57.50 & 55.00 & \begin{tabular}{|l|}
45.00 \\
\end{tabular} & 65.00 & \begin{tabular}{|l|}
77.27 \\
\end{tabular} & 40 \\
\hline Trachea & 100.00 & 100.00 & 100.00 & 100.00 & 100.00 & 100.00 & 100.00 & 40 \\
\hline LMB & 100.00 & 100.00 & 100.00 & 100.00 & 100.00 & 100.00 & 100.00 & 40 \\
\hline LUL & 100.00 & 100.00 & 100.00 & 100.00 & 100.00 & 100.00 & 100.00 & 40 \\
\hline LB4+5 & 97.50 & 95.00 & 96.25 & 97.50 & \begin{tabular}{|l|}
97.50 \\
\end{tabular} & 95.00 & \begin{tabular}{|l|}
97.44 \\
\end{tabular} & 40 \\
\hline LLB & 100.00 & 100.00 & 100.00 & 100.00 & 100.00 & 100.00 & \begin{tabular}{|l|}
100.00 \\
\end{tabular} & 40 \\
\hline$\overline{\mathrm{RMB}}$ & 100.00 & 100.00 & 100.00 & 100.00 & \begin{tabular}{|l|}
100.00 \\
\end{tabular} & 100.00 & 100.00 & 40 \\
\hline RUL & 90.00 & 90.00 & 90.00 & 90.00 & 85.00 & 90.00 & 100.00 & 40 \\
\hline BronchInt & 100.00 & 100.00 & 100.00 & 100.00 & 100.00 & 100.00 & 100.00 & 40 \\
\hline RLL & 92.50 & 95.00 & 93.75 & 97.50 & 97.50 & 90.00 & \begin{tabular}{|l|}
94.87 \\
\end{tabular} & 40 \\
\hline Avg segment & 71.57 & 73.92 & 72.74 & 71.00 & \begin{tabular}{|l|}
72.63 \\
\end{tabular} & 76.00 & \begin{tabular}{|l|}
83.49 \\
\end{tabular} & 39.5 \\
\hline Avg total & 79.70 & 81.32 & 80.51 & 79.48 & \begin{tabular}{|l|}
80.43 \\
\end{tabular} & 82.59 & \begin{tabular}{|l|}
88.35 \\
\end{tabular} & 39.6 \\
\hline
\end{tabular}


Labeling Results. The labeling was implemented in MATLAB, using tree distance computations implemented in Java. For airway trees with 150 branches on average, the whole labeling takes, roughly, 5 minutes per tree running on a single $2.40 \mathrm{GHz}$ processor on a laptop with 8 GB RAM.

The labeling was tested in a leave-one-patient-out fashion. Thus for each airway, the training set was made up of 38 airway trees from other patients, with each tree labeled separately by the two medical experts, giving a total of 76 training airway trees. The results of the labeling are shown in table 1, along with a comparison of the two expert labelings.

In order to test reproducibility of the expert and automatic labels, the two CT scans of each subject were registered using the image registration method described in $\underline{3}$, and the labeled airway trees were compared in a common coordinate system. Expert 1, Expert 2 and the automatic algorithm reproduced 14.0, 15.1 and 15.2 labels per subject on average. The automatic algorithm was not significantly different from the average expert ( $p=0.51$ in a paired t-test).

On average, the automatic labeling agreement with an expert is $72.8 \%$ on the segment branches, which is not significantly different from the average interexpert agreement of $71.0 \%$ ( $p=0.75$ in a paired t-test). Fig. [ 4 shows labeling performance stratified by COPD stage. Spearman's correlation test shows no significant correlation between the average agreement with an expert and the severity of $\operatorname{COPD}(\rho=-0.22, p=0.18)$.

\section{Discussion and Conclusion}

Higher percentages are reported in the literature: $97.1 \%, 90 \%, 83 \%$ on all branch labels in [10, high dose CT], [1], [5]; 77\% on segment labels [5]. These methods use fewer than 20 segment labels and/or more intermediate (easier) labels, and reject uncertain labels using a threshold. On average, only $71 \%, 93 \%$ and $83 \%$ of the given label set [10, [11, [5] and at most $77 \%$ of the given segment label set [5] are assigned, whereas we almost always assign all 20 segment labels. The $97.1 \%$ success rate 10 is among branches that have been labeled identically by three experts. Taking unassigned labels into account in [5, 11,10, these do not appear to perform better, and do not test reproducibility. We, on the other hand, perform just as well and reproducibly as a medical expert on our dataset.

Our evaluation, which is thorough compared to previous work, gives detailed insight into the difficulties of the labeling problem. It is noteworthy that the experts and the automatic method perform well in different parts of the airway tree. In particular, the automatic method is far more reproducible in parts of the airway tree where the experts have difficulties, e.g., the lower left lobe (L7-L10).

The fact that the method as presented always assigns all segment labels if possible, makes it sensitive to missing branches and increases our false positive rate on difficult branches. This could be tackled by introducing label probabilities based on the geodesic airway tree distances, and thus assigning fewer labels. 
The hierarchical scheme of Fig. 3 may cause difficulties with rare topologies. This could be handled by a more refined hierarchical labeling scheme, particularly one informed by an analysis of where the experts performed better. The labeling is sensitive to mistakes made above the segment level. This could be improved by label probabilities; however, the algorithm rarely makes such mistakes.

We present a new supervised method for anatomical branch labeling of airway trees, based on geodesic distances between airway trees in tree-space. Using the distances, the algorithm evaluates how well a suggested branch labeling fits with a training set of labeled airway trees, and chooses the optimal labeling. The labeling performance is robust in patients with COPD, and is comparable to that of two experts in pulmonary medicine. As it only uses branch centerlines and tree topology, we expect it to generalize to other datasets. Its reproducibility and robustness in patients with COPD makes it highly suitable for clinical use.

Acknowledgement. This research was supported by the Lundbeck Foundation; AstraZeneca; The Danish Council for Strategic Research; Netherlands Organisation for Scientific Research; Centre for Stochastic Geometry and Advanced Bioimaging, funded by the Villum Foundation. M.O. was funded by a FieldsOntario Postdoctoral Fellowship.

\section{References}

1. Billera, L.J., Holmes, S.P., Vogtmann, K.: Geometry of the space of phylogenetic trees. Adv. in Appl. Math. 27(4), 733-767 (2001)

2. Feragen, A., Lo, P., Gorbunova, V., Nielsen, M., Dirksen, A., Reinhardt, J.M., Lauze, F., de Bruijne, M.: An airway tree-shape model for geodesic airway branch labeling. In: MICCAI WS on Math. Found. Comp. Anat. (2011)

3. Gorbunova, V., Sporring, J., Lo, P., Loeve, M., Tiddens, H., Nielsen, M., Dirksen, A., de Bruijne, M.: Mass preserving image registration for lung CT. MedIA (2012)

4. Hasegawa, M., Nasuhara, Y., Onodera, Y., Makita, H., Nagai, K., Fuke, S., Ito, Y., Betsuyaku, T., Nishimura, M.: Airflow Limitation and Airway Dimensions in Chronic Obstructive Pulmonary Disease. Am. J. Respir. Crit. Care Med. 173(12), 1309-1315 (2006)

5. Lo, P., van Rikxoort, E.M., Goldin, J., Abtin, F., de Bruijne, M., Brown, M.: A bottom-up approach for labeling of human airway trees. In: MICCAI Int. WS. Pulm. Im. Anal. (2011)

6. Owen, M., Provan, J.S.: A fast algorithm for computing geodesic distances in tree space. ACM/IEEE Trans. Comp. Biol. Bioinf. 8, 2-13 (2011)

7. Pedersen, J., Ashraf, H., Dirksen, A., Bach, K., Hansen, H., Toennesen, P., Thorsen, H., Brodersen, J., Skov, B., Døssing, M., Mortensen, J., Richter, K., Clementsen, P., Seersholm, N.: The Danish randomized lung cancer CT screening trial. Overall design and results of the prevalence round. J. Thor. Onc. 4(5), 608-614 (2009)

8. Petersen, J., Nielsen, M., Lo, P., Saghir, Z., Dirksen, A., de Bruijne, M.: Optimal Graph Based Segmentation Using Flow Lines with Application to Airway Wall Segmentation. In: Székely, G., Hahn, H.K. (eds.) IPMI 2011. LNCS, vol. 6801, pp. 49-60. Springer, Heidelberg (2011) 
9. Rabe, K.F., Hurd, S., Anzueto, A., Barnes, P.J., Buist, S.A., Calverley, P., Fukuchi, Y., Jenkins, C., Rodriguez-Roisin, R., van Weel, C., Zielinski, J.: Global strategy for the diagnosis, management, and prevention of chronic obstructive pulmonary disease: Gold executive summary. Am. J. Respir. Crit. Care Med. 176(6), 532-555 (2007)

10. Tschirren, J., McLennan, G., Palágyi, K., Hoffman, E.A., Sonka, M.: Matching and anatomical labeling of human airway tree. TMI 24(12), 1540-1547 (2005)

11. van Ginneken, B., Baggerman, W., van Rikxoort, E.M.: Robust Segmentation and Anatomical Labeling of the Airway Tree from Thoracic CT Scans. In: Metaxas, D., Axel, L., Fichtinger, G., Székely, G. (eds.) MICCAI 2008, Part I. LNCS, vol. 5241, pp. 219-226. Springer, Heidelberg (2008) 